\title{
ENERGY EFFICIENT COVERAGE PROBLEMS IN WIRELESS Ad Hoc SENSOR NETWORKS
}

\author{
D.G.Anand ${ }^{1}$, Dr.H.G.Chandrakanth ${ }^{2}$ and Dr.M.N.Giriprasad ${ }^{3}$ \\ ${ }^{1,2}$ Sri Krishna Institute of Technology, Bangalore, Karnataka, \\ ${ }^{3}$ Jawaharlal Nehru Technological University College of Engineering, \\ Pulivendula, Andra Pradesh
}

\begin{abstract}
Coverage is a typical problem in wireless sensor networks to fulfil the issued sensing tasks. In general, sensing coverage represents how well an area is monitored by sensors. The quality of a sensor network can be reflected by the levels of coverage and connectivity that it offers. The coverage problem has been studied extensively, especially when combined with connectivity and energy efficiency. Constructing a connected fully covered, and energy efficient sensor network is valuable for real world applications due to the limited resources of sensor nodes. In this paper, we survey recent contributions addressing energyefficient coverage problems in the context of static WASNs, networks in which sensor nodes do not move once they are deployed and present in some detail of the algorithms, assumptions, and results. A comprehensive comparison among these approaches is given from the perspective of design objectives, assumptions, algorithm attributes and related results.
\end{abstract}

\section{KEYWORDS}

Algorithms, Design, Performance, Additional Key Words and Phrases: ad hoc network, coverage, connectivity, energy conservation, power control, sensor network, wireless network, WASN, QoS

\section{INTRODUCTION}

The rapid progress of wireless communication and embedded micro-sensing MEMS technologies has made wireless sensor networks possible. Such environments may have many inexpensive wireless nodes, each capable of collecting, storing, and processing environmental information, and communicating with neighbouring nodes. [1] In general, the wireless sensor networks can contain hundreds or even thousands of sensing nodes. It is desirable to make these nodes as energy-efficient as possible and to rely on their large numbers in order to obtain high quality results. Since sensors may be spread in an arbitrary manner; a fundamental issue in a wireless sensor network is to ensure coverage and connectivity.

In the literature, this problem has been formulated in various ways. Coverage can be regarded as a metric to evaluate the quality of service (surveillance) provided by the network [29]. Between a given pair of points in the sensing field, some works focus on finding a path connecting these two points which is best or worst monitored by sensors when an object traverses along the path. An important research issue in wireless ad hoc sensor network is the coverage problem. Sensing coverage characterizes the monitoring quality provided by a sensor network in a designated region. Different applications require different degrees of sensing coverage. While some applications may only require that every location in a region be monitored by multiple nodes, and distributed tracking and classification [28] requires even higher degrees of coverage. The coverage requirement for a sensor network also depends on the number of faults that must be tolerated. The coverage requirement may also change after a network has been deployed, for instance, due to changes in application modes or environmental conditions. For example, a surveillance sensor network may initially maintain a low degree of coverage required for distributed detection. After an intruder is detected, however, the region in the vicinity of the 
intruder must reconfigure itself to achieve a higher degree of coverage required for distributed tracking.

Energy conservation is a critical issue in sensor networks because replacement of battery is costly and even infeasible in some applications, such as battle field surveillance. A frequently used method to conserve energy is scheduling, where a minimum number of sensor nodes are activated to satisfy the $K$-coverage requirement and the remaining nodes are set to sleep for conserving the energy.

In this paper, we survey the existing researches on the coverage problem describing and comparing algorithms that have been presented in the literature.

The paper continues with Section 2 that describes the approaches with different design objectives on the coverage problem including the scope, assumptions, and algorithms. In Section 3, these approaches are compared according to different characteristics of design objectives, assumptions, algorithm attributes, and related results, followed by concluding remarks in section 4 .

\section{DESIGN OBJECTIVES}

This section describes the approaches with different design objectives on the coverage problem including the scope, assumptions, and algorithms.

In [22] authors explain the coverage in sensor networks as, two seemingly contradictory, yet related viewpoints of coverage exist: worst and best case coverage. In worst-case coverage, attempts are made to quantify the quality of service by finding areas of lower observability from sensor nodes and detecting breach regions. In best-case coverage, finding areas of high observability from sensors and identifying the best support and guidance regions are of primary concern.

We surveyed existing methods and their contributions which address various research objectives in the coverage problem .In the following sub-sections; we will present in some detail the algorithms or solutions, their assumptions, and results.

\section{A. Coverage Configuration Protocol (CCP)}

Wang et al [19] proposed the Coverage Configuration Protocol that can provide different degrees of coverage and meanwhile maintain communication connectivity is presented. If the communication ranges are less than twice the sensing ranges, proposes to integrate $\mathrm{CCP}$ with SPAN to provide both sensing coverage and communication connectivity. A similar result is also drawn in, and thus only the coverage problem is addressed. Coverage Configuration experiment is performed on the Coverage Simulator (CS) compares the performance of CCP to the Ottawa protocol described in[20]. Similar to CCP, the Ottawa protocol is a decentralized protocol designed to preserve coverage while turning off redundant nodes to conserve energy in a sensor network. Simulation results reported in [20] also demonstrated that this protocol can provide better coverage than the PEAS protocol which is designed to control density rather than coverage. The Ottawa protocol and CCP utilize different eligibility rules. The main advantage of CCP over the Ottawa protocol lies in its ability to configure the network to the specific coverage degree requested by an application, while the Ottawa protocol does not support different coverage configurations. In addition, the experimental results show that even when only 1-coverage is required, CCP results in a smaller number of active nodes and hence leads to more energy conservation than the Ottawa protocol. All the results in this section are based on five runs with different random network topologies. The region used for testing in Experiment is $50 \mathrm{~m} \times 50 \mathrm{~m}$ if not specified otherwise, and the sensing range is $10 \mathrm{~m}$ for all nodes.

Next, the authors evaluate the capability of several protocols in terms of providing integrated coverage and connectivity configuration in NS-2. The following protocols are compared:

- SPAN, obtained from MIT (http://www.pdos.lcs.mit.edu/span/);

- CCP, implemented by replacing the SPAN's coordinator eligibility rule with CCP's; 
- SPAN+CCP, implemented by combining the eligibility rules of SPAN and CCP,CCP-2Hop, implemented by adding the locations of a node's neighbouring coordinators in its HELLO message - SPAN+CCP-2Hop, SPAN+CCP with extended HELLO messages as in CCP-2Hop.

\section{B. OGDC (Optimal Geographical Density Control).}

Zhang and Hou [18] present the protocol OGDC (Optimal Geographical Density Control). According to the authors, this is a full distributed and local search algorithm based on the following: if the communication (or radio) range is at least twice of the sensing range, then a complete coverage of a convex area implies the connectivity among the working set of nodes. Based on this assumption, authors present a set of optimality conditions under which a subset of working sensor nodes can be chosen for full coverage and also propose an algorithm that assures these conditions when the network has a high density and each node knows its own position in the network area.

\section{PEAS (Probing Environment and Adaptive Sleeping).}

Ye et al [15] present the algorithm PEAS (Probing Environment and Adaptive Sleeping). PEAS consists of two algorithms, which determine (1) which sensor nodes should work and how a wake-up sensor node makes the decision of whether going back to sleep, and (2) how the average sleep time of the sensor nodes is dynamically adjusted to keep a relatively constant wake-up rate.

Sleeping nodes wake up once in a while to broadcast a probing message over a certain range and to replace any failed working node. The probing range can be adjusted to achieve different levels of coverage overlap, but it also cannot ensure complete coverage [14].

\section{PECAS (Probing Environment and Collaborating Adaptive Sleeping)}

PECAS is proposed by Gui and Mohapatra. It is an extension of the PEAS protocol proposed in [10]. It has the same en environment probing mechanism as PEAS, but it does not let working nodes stay awake indefinitely. The designers argue that "the failure of nodes (due to energy depletion) may cause partitioning of the network or isolation of nodes. It is desirable to balance energy consumption among the neighbouring nodes." Therefore, a working node in PECAS will go back to sleep after a specified period of time. It also advertises the remaining working time in its reply messages to its neighbours' probe messages. In this way, a working neighbour who decides to enter the off-duty -mode can schedule itself to wake up before this node goes to sleep, thus preventing the occurrence of blind spots.

\section{E. ASCENT (Adaptive Self-Configuring sEnsor Networks Topologies)}

In, Cerpa and Estrin proposed using sensors' local measurements to automatically configure network topology in a high density sensor network. The goal is to maintain a certain data delivery ratio while allowing redundant sensors to stay asleep in order to conserve energy. Achieving this goal requires configuring the network to the right level of connectivity; it cannot be too low to hamper data delivery, but it cannot be too high either since neighbouring nodes might interfere with each other leading to a high collision rate. The approach adopted by ASCENT is to let sensors measure their connectivity as well as their data loss rate and activate their neighbours based on these local measurements.

ASCENT is similar to PEAS in several ways. First, ASCENT assumes that there is a high density of sensor nodes. Second, after the sensors are activated, the they never go back to sleep - fairness in energy consumption is not an important design issue here. Third, both are decentralized mechanisms that allow sensors to use locally measured information to adjust network topology. However, unlike PEAS, ASCENT does not guarantee network connectivity 
in any sense (the network could be partitioned), although the delivery of data indicates that there is a certain level of connectivity.

In summary, ASCENT allows sensors to automatically adapt their connectivity to achieve a required le level of data delivery ratio. It has the following wing major characteristics: (1) nodes do not need accurate location information; (2) there is no periodic message overhead for neighbour discovery; (3) nodes maintain per-neighbour -state to keep track of the number of active neighbours; (4) working nodes never go back to sleep, which may result in unfair air energy consumption[11].

\section{F. Sleep + Power Control (PC) Protocol}

This work [16] is also a significant extension of authors' earlier work [Huang and Tseng 2003; Huang et al. 2004], which addresses how to determine the level of coverage of a given sensor network, but does not consider the network connectivity issue. This work is the first work allowing an arbitrary relationship between sensing ranges and communication distances of sensor nodes. They develop decentralized solutions for determining, or even adjusting, the levels of coverage and connectivity of a given network. Adjusting levels of coverage and connectivity is necessary when sensors are overly deployed, and we approach this problem by putting sensors to sleep mode and tuning their transmission powers. This results in prolonged network lifetime. Both Sleep and Sleep+PC protocols can significantly reduce the rate that sensors fail. Overall, Sleep+PC perform the best. The lifetime of the naive protocol is around $150 \mathrm{sec}$. The lifetime of CCP+SPAN is around $200 \mathrm{sec}$. The Sleep and Sleep+PC protocols can significantly prolong network lifetime to around 340 and $410 \mathrm{sec}$. , basically, these schemes can perform better when sensors have larger communication ranges. This is because sensors with larger transmission ranges can find more neighbours, collect more necessary information needed for making sleeping and power-controlling decisions, and thus have higher chance to go to sleep and/or shrink their powers. we further test under different coverage and connectivity requirements. Around 1 to 2 times more lifetime can be seen when comparing Sleep+PC to $\mathrm{CCP}+\mathrm{SPAN}$.

\section{G. Coverage Aware Sensor Engagement (CASE)}

In [21], a novel sensor network coverage maintenance protocol called Coverage Aware Sensor Engagement (CASE) was designed to efficiently maintain the required degree of sensing coverage by activating a small number of sensors while putting the others in sleep mode. CASE schedules active/inactive sensing states of a sensor according to the sensor's contribution to the network sensing coverage, which is quantitatively measured by a metric called coverage merit". By activating sensors with relatively large coverage merit and deactivating those with small coverage merit, CASE effectively achieves energy conservation while maintaining sufficient sensor network coverage.

\section{H. Geographical adaptive fidelity (GAF) strategy}

It is based on [16] a subdivision of the network deployment region into an appropriate number of non-overlapping square cells. The cells are used to identify equivalent nodes. All the nodes lying on the same cell are proved to be routing equivalent. An election algorithm is periodically executed to elect the leader in every cell.

Geographical adaptive fidelity (GAF) algorithm reduces energy consumption in ad hoc wireless networks. GAF conserves energy by identifying nodes that are equivalent from a routing perspective and then turning off unnecessary nodes, keeping a constant level of routing fidelity. GAF moderates this policy using application- and system-level information; nodes that source or sink data remain on and intermediate nodes monitor and balance energy use. GAF is independent of the underlying ad hoc routing protocol. Analysis and simulation studies of GAF show that it can consume $40 \%$ to $60 \%$ less energy than an unmodified ad hoc routing protocol. 
GAF increases network lifetime proportionally to node density. More generally, GAF is an example of adaptive fidelity, a technique proposed for extending the lifetime of self-configuring systems by exploiting redundancy to conserve energy while maintaining application fidelity [7]

Thai et al. [23] proposed an $O(\log n)$-approximation distributed algorithm for the target coverage problem. They organize the sensors into non-disjoint cover sets such that each set completely covers all the targets. The authors reduce the target coverage problem to the domatic number problem in directed graphs.

Cardei et al. [24] study the energy efficient target coverage problem in wireless sensor networks. They consider a number of targets with known locations that needed to be continuously observed by the sensors. Their objective is to have each location in the physical space of target within the sensing range of at least one sensor. They proposed an efficient method to extend the sensor network lifetime by dividing the sensor nodes into a number of sets, such that each set completely covers all the targets, and these sensor sets are scheduled to alternate between active state and sleep state. However, their proposed linear programming and greedy heuristics are centralized, which is not desirable in wireless sensor networks.

\section{SENSOR COVERAGE PROBLEMS AND DESIGN CHOICES}

We first give an overview of design choices for sensor coverage problems and then discuss related work in other fields.

\section{A. Our Design Goals and Assumptions}

Sensors have size, weight, and cost restrictions, which impact resource availability. They have limited battery resources and limited processing and communication capabilities. As replacing the battery is not feasible in many applications, low power consumption is a critical factor to be considered, not only in the hardware and architectural design, but also in the design of algorithms and network protocols at all layers of the network architecture. Therefore maximizing the network lifetime is an important network design objective.

In order to prolong the whole sensor network lifetime and maintain the area coverage problem, we should have energy-efficient Coverage Preserving Protocol to select the set of active sensor nodes. The protocols are designed to achieve several purposes and put emphasis on the following main goals that we perform:

(a) Detect whether the monitoring region is completely covered or not.

(b) Detect the degree of the coverage in entire monitoring region.

(c) Minimize the total number of active sensor nodes.

(d) Diminish the total amount of energy consumption.

(e) Support an extension to satisfy the multiple degree of coverage requirement.

(f) Support an extension to realize the probabilistic coverage model

(g) Support the desired communication/computation power usage ratio

The coverage algorithms proposed are either centralized or distributed. In distributed algorithms, the decision process is decentralized. By distributed and localized algorithms, we refer to a distributed decision process at each node that makes use of only neighbourhood information, within a constant number of hops. Because the WASN has a dynamic topology and needs to accommodate a large number of sensors, the algorithms and protocols designed should be distributed and localized, in order to better accommodate a scalable architecture. 


\section{B. Coverage Problems}

The most discussed coverage problems in literature can be classified in the following types: area coverage, point coverage and barrier coverage. Based on the subject to be covered (area versus discrete points), different problems can be formulated considering the following design choices:

Objective of the Problem: Coverage, maximum network lifetime or minimum number of sensors.

(1) Random Deployment: Suppose the entire sensor nodes are deployed randomly in regular (rectangle) monitoring region and deployed in uniform distribution. In addition, all nodes are static without any moving.

(2) Location Information Known: Each sensor node knows its own location e.g. through GPS technique or other localization protocols [4], and each node exchanges own location information with its 1-hop neighboring nodes.

(3) Uniform Power Decay: Every node is expected to uniform node lifetime, and the power decline also uniformly with time.

(4) Circular Sensing Model: The sensing area of every node is assumed circular. Every node has the same sensing range $\left(R_{\mathrm{s}}\right)$ and communication range $\left(R_{\mathrm{c}}\right)$, i.e., a homogeneous network.

(5) $\boldsymbol{R}_{c} \geqq \mathbf{2} \boldsymbol{R}_{s}$ Relationship: The communication range is greater than two times of the same sensing range. This is sufficient condition for coverage to imply to connectivity, i.e., for a set of sensors that sufficiently cover a monitoring region, the communication graph is connected if $R_{\mathrm{c}} \geqq 2 R_{\mathrm{s}}$.

\section{Comparison of Algorithm Attributes}

The attributes of each algorithm are discussed from the following perspectives: distribution, localization, and coverage degree. A distributed algorithm implements the scheduling mechanism in a decentralized way. There is no centre node which decides the status of other nodes; instead, the decision is made by every node. Most protocols use distributed algorithms, such as CCP, OGDC \& Sleep + PC construction algorithm. In a localized algorithm, the scheduling decision can be made based on one hop neighbourhood information.

\section{Table1}

Comparisons of Design Results

\begin{tabular}{|l|l|l|l|}
\hline & Area coverage & Connectivity & Energy \\
\hline CCP & Yes & Yes & Yes \\
\hline OGDC & Yes & Yes & Yes \\
\hline Sleep+PC & Yes & Yes & Yes \\
\hline
\end{tabular}

\section{CONCLUSION}

In this paper we have identified a comprehensive list of Coverage related issues associated with Wireless Sensor Networks. We have also discussed some popular protocols implementing these issues in part or as a whole. Wireless sensor networks are more than just a specific form of ad hoc networks. Recent advanced hardware technologies result in more powerful sensors as small as a few millimetres volume. The main drawbacks are still energy constraints \& coverage 
problem, which reflects the quality of service that can be provided by a particular sensor network. In this paper, we survey recent contributions addressing energy-efficient coverage problems in the context of static WASNs, networks in which sensor nodes do not move once they are deployed and present in some detail of the algorithms, assumptions, and results. A comprehensive comparison among these approaches is given from the perspective of design objectives, assumptions, algorithm attributes and related results.

Most recent works on the sensor coverage problem are still limited to theoretical study, where only centralized solutions are given. As wireless sensor networks are still young research fields; much activity is still on-going to solve many open issues. This field is surely going to give us tremendous opportunity to change the way we perceive the world today

\section{REFERENCES}

[1] Chi-Fu Huang, Yu-Chee Tseng, and Hsiao-Lu Wu, "Distributed Protocols for Ensuring Both Coverage and Connectivity of a Wireless Sensor Network" of National Chiao-Tung University, ACM Journal Name, Vol. V, No. N, M 20YY, Pages 1-22.

[2].Guoliang Xing, Xiaorui Wang, Yuanfang Zhang, Chenyang Lu,Robert Pless, And Christopher Gill" Integrated Coverage and Connectivity Configuration for Energy Conservation in Sensor Networks" Washington University in St. Louis- in ACM Transactions on Sensor Networks, Vol. 1, No. 1, August 2005.pages 36-72.

[3] Mihaela Cardei and Jie Wu, Department of Computer Science and Engineering "EnergyEfficient Coverage Problems in Wireless Ad Hoc Sensor Networks" Florida Atlantic University, Boca Raton, FL 33431

[4]. Jie Chen and Xenofon Koutsoukos, Vanderbilt University "Survey on Coverage Problems in Wireless Ad Hoc Sensor Networks"

[5]. Chi-Fu Huang, Yu-Chee Tseng, and Hsiao-Lu Wu "Distributed Protocols for Ensuring Both Coverage and Connectivity of a Wireless Sensor Network "National Chiao-Tung University, ACM Journal Name, Vol. V, No. N, M 20YY.

[6] V. Raghunathan, C. Schurgers, S. Park, and M. B. Srivastava, Energy-Aware Wireless Microsensor Networks, IEEE Signal Processing Magazine, 19 (2002) 40-50.

[7] Ya Xu, John Heidemann, Deborah Estrin "Geography-informed energy conservation for Ad Hoc routing"

[8] F. Ye, G. Zhong, and J. Cheng e L. Zhang. Peas: A robust energy conserving protocol for long-lived sensor networks. In Proceedings of the 23rd International Conference on Distributed Computing Systems (ICDCS03), pages 28-37, 2003.

[9] C. Gui and P. Mohapatra. Power conservation and quality of surveillance in target tracking sensor networks.. In Proceedings of the 10th Annual International Conference on Mobile Computing and Networking (Mobicom '04), pages 129-143, 2004.

[10] F. Ye, G. Zhong, J. Cheng, S. Lu, and L. Zhang. Peas: A robust energy conserving protocol for long-li lived sensor networks. In Proceedings of the 23r 23rd International Conference on Distributed Computing Systems (ICDCS '03), pages 28-37, 2003. 
[11] Lan Wang and Yang Xiao: A Survey of Energy -Efficient Scheduling Mechanisms in Sensor Networks , Department of Computer Science, The University of Memphis TN 38152 USA

[12]A. Cerpa and D. Estrin. Ascent: Adaptive self-configuring sensor networks topologies. In Proceedings of IEEE INFOCOM 2002, New York, NY, June 2002.

[13]Anusha PB, Darshana Wagh," Placement, coverage and connectivity properties of sensor networks" 2008, Indian Institute of Technology, Kanpur

[14] Vyacheslav Zalyubovskiy, Adil Erzin, Sergey Astrakov and Hyunseung, Energy-efficient Area Coverage by Sensors with Adjustable Ranges, Sensors ,2009, 9, 2446-2460

[15] F. Ye, G. Zhong, J. Cheng, S. Lu, and L. Zhang, "PEAS: A Robust Energy Conserving Protocol for Long-Lived Sensor Networks," Proc. Of the 23rd International Conference on Distributed Computing Systems (ICDCS'03), Page(s): 28 - 37, May. 2003.

[16].Chi-Fu Huang, Yu-Chee Tseng, and Hsiao-Lu Wu, "Distributed Protocols for Ensuring Both Coverage and Connectivity of a Wireless Sensor Network" ACM Journal Name, Vol. V, No. N, M 20YY, Pages 1-22.

[17].HUANG, C.-F. AND TSENG, Y.-C. 2003. The coverage problem in a wireless sensor network. In ACM Int'l Workshop on Wireless Sensor Networks and Applications (WSNA).115121.

[18] H. Zhang and J. C. Hou, Maintaining Sensing Coverage and Connectivity in Large Sensor Networks, Technical Report UIUC, UIUCDCS-R- 2003-2351 (2003).

[19]. X. Wang, G. Xing, Y. Zhang, C. Lu, R. Pless, and C. D. Gill, Integrated Coverage and Connectivity Configuration in Wireless Sensor Networks, accepted to the First ACM Conference on Embedded Networked Sensor Systems (2003).

[20]D. Tian and N. D. Georganas, "A coverage-preserving node-scheduling scheme for large wireless sensor networks," Processing of ACM wireless sensor network and application workshop 2002, Sept. 2002.

[21] J. Lu, L. Bao, and T. Suda, "Coverage-aware sensor engagement in dense sensor networks," Journal of Embedded Computing, vol. 3, no. 1, pp. 3-18, 2009.

[22]. Seapahn Meguerdichian, Farinaz Koushanfar, Miodrag Potkonjak, Mani B. Srivastava" Coverage Problems in Wireless Ad-hoc Sensor Networks" IEEE TRANSACTIONS ON MOBILE COMPUTING, VOL. 3, NO. 4, OCTOBER-DECEMBER 2004

[23] Mihaela Cardei, Jie Wu, and Mingming Lu, "Improving network lifetime using sensors with adjustable sensing ranges," in International Journal of Sensor Networks, 2006, pp. 41-49.

[27] Mihaela Cardei, My T. Thai, Yingshu Li, and Weili Wu, LEnergy-efficient target coverage in wireless sensor networks," in IEEE INFOCOM, March 2005, vol. 3, pp. 1976-1984.

[28] LI, D., WONG K., HU, Y.H. AND SAYEED A. 2002. Detection, classification and tracking of targets in distributed sensor networks, IEEE Signal Processing Magazine 19, 2, 17 29 
[29].Nor Azlina Ab. Aziz, Kamarulzaman Ab. Aziz, and Wan Zakiah Wan Ismail" Coverage Strategies for Wireless Sensor Networks" World Academy of Science, Engineering and Technology 2009, pp 145-150

\section{Authors:}

D. G. Anand is native of Madikeri, Karnataka, India. He received his B.E Degree from AIT, Chikamagalore, Mysore University and ME from UVCE Bangalore University, Bangalore and currently he is persuing his PhD from Jawaharlal Nehru Technological University Anantapur, Andra Pradesh. Presently he is serving as Professor and HOD in the department of ECE at Sri Krishna Institute Of Technology, Bangalore. His areas of interest are wireless communication, sensor networks.

(dg_anand2003@ sifymail.com)

Dr.M.N.Giri Prasad is native of Hindupur town of Anantapur District of Andhra Pradesh, India. He received B.Tech degree from J.N.T University College of Engineering, Anantapur, Andhra Pradesh, India in 1982, M.Tech degree from Sri Venkateshwara University, Tirupati, Andhra Pradesh, India in 1994 and $\mathrm{PhD}$ degree from J.N.T University, Hyderabad, Andhra Pradesh, India in 2003. Presently he is working as Professor and Head, department of Electronics and Communication at J.N.T University College of Engineering, Pulivendula, and

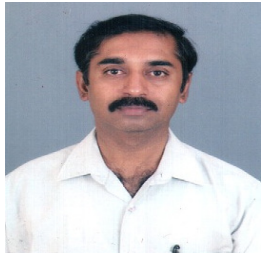
Andhra Pradesh, India.

His research areas are Wireless Communications and Biomedical Instrumentation. He is a member of ISTE, IE \& NAFEN

(e-mail:mahendran_gp@rediffmail.com).

Dr. H. G. Chandrakanth is native of Bangalore, Karnataka, India. He received B.E Degree from UVCE, Bangalore University, Bangalore, India in 1991, MSEE from Southern Illinois University Carbondale, USA in 1994 and $\mathrm{PhD}$ from Southern Illinois University Carbondale, USA in 1998. Presently he is working as Principal in Sri Krishna Institute Of Technology, Bangalore.

(ckgowda@hotmail.com)

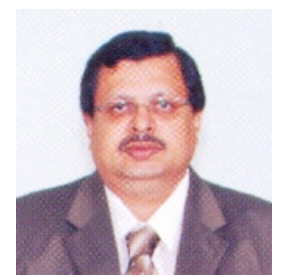

